Ann. Génét. Sél. anim., I97I, 3 (4), 433-448.

\title{
ÉTUDE DU TAUX D'HYDROXYPROLINE D'UN MUSCLE ET DE SES RELATIONS AVEC CERTAINES CARACTÉRISTIQUES DE COMPOSITION CORPORELLE DANS LES RACES LARGE WHITE ET DE PIETRAIN
}

\author{
P. SELLIER et R. BOCCARD \\ avec la collaboration technique de Nathalie Boutrer, \\ P. Dando et Georgette Nicolas \\ Station de Génétique quantitative et appliquée, \\ Centre national de Recherches zootechniques, I. N. R. A., \\ 78 - Jouy-en-Josas \\ Station de Recherches sur la Viande, \\ Centre de Recherches de Clermont-Ferrand, I. N. R. A., \\ 63 - Theix par Saint-Genès-Champanelle
}

\section{RÉ:SUMÉ}

En deux séries d'abattage (au poids de $95 \mathrm{~kg}$ ), les carcasses de 197 porcs de Piétrain, $\mathrm{r} 69$ porcs de race Large White et 267 porcs Piétrain $\times$ Large White ont été découpées et 13 mesures de carcasse ont été considérées : poids des différents morceaux, longueur, trois mesures d'épaisseur du lard dorsal. Par ailleurs, le taux d'hydroxyproline du muscle Triceps brachii caput laterale a été déterminé : il est exprimé par le rapport de l'azote de cet acide aminé à l'azote total du muscle (en p. I ooo).

Le taux d'hydroxyproline est plus élevé $(\mathrm{P}<0,05)$ chez les femelles que chez les mâles castrés. Les différences entre les trois groupes génétiques sont hautement significatives $(\mathrm{P}<0, \mathrm{O})$ : 3,789 $\pm 0,033 \mathrm{chez}$ les porcs de Piétrain, 3,958 $\pm 0,037 \mathrm{chez}$ les porcs Piétrain $\times$ Large White, 4,295 $\pm 0,036 \mathrm{chez}$ les porcs Large White. Une analyse discriminante a été réalisée, à partir des I4 variables considérées, sur les échantillons Piétrain et Large White de la deuxième série d'abattages, la sélection progressive des variables étant basée sur la statistique $\mathrm{D}^{2}$ de Mahalanobis. Le taux d'hydroxyproline du muscle étudié est, avec la longueur de carcasse, l'épaisseur de lard au niveau du rein et le poids de la longe, l'un des principaux critères de discrimination entre le type Piétrain, à musculature hypertrophiée, et le type Large White. Cependant aucune relation simple ne semble exister entre ce caractère et les caractéristiques de conformation et de composition corporelle à l'intérieur de l'une et l'autre race 


\section{INTRODUCTION}

La race de Piétrain a fait l'objet de nombreuses études depuis une quinzaine d'années et il est clairement établi qu'elle se distingue nettement des autres races porcines européennes par sa conformation et sa composition corporelle. L'hypertrophie musculaire, surtout apparente au niveau du membre postérieur, est l'une des caractéristiques les plus constantes de ce "type Piétrain ", comme le montrent les résultats de dissection de carcasse obtenus par MESLE et al. (I959), BLENDL (I965) et Dumont et al. (I969). Le déterminisme héréditaire de 1'hypertrophie musculaire du porc de Piétrain a été étudié par OlLIVIER (I968) : ses résultats suggèrent que les gènes responsables sont en nombre limité.

Récemment des études ont été entreprises en France dans le but de décrire les principales caractéristiques du muscle chez le porc de Piétrain, notamment l'importance relative du tissu conjonctif, et de préciser éventuellement l'origine de l'hypertrophie musculaire dans cette race. Dans une première étude, Boccard (r968) a montré que la teneur du muscle en hydroxyproline, acide aminé caractéristique du collagène, est significativement plus faible en race de Piétrain qu'en race Large White, l'ampleur de la différence dépendant dans une large mesure du muscle étudié. Sur un plus grand nombre d'animaux et en considérant un seul muscle (Triceps brachii caput laterale), SELLIER et BOCCARD (I969) ont retrouvé une différence hautement significative entre les 2 races pour le taux d'hydroxyproline; 1'ensemble de leurs résultats indiquait par ailleurs qu'à l'intérieur des races considérées, le degré de développement musculaire et le taux d'hydroxyproline du muscle étudié apparaissaient comme deux variables pratiquement indépendantes. Parallèlement à cette voie de recherche, l'étude comparée de la trame conjonctive de certains muscles dans les races Large White et de Piétrain a été abordée sur le plan de l'analyse histologique (Dumon' et Schmitr, I970). L'étude du taux d'hydroxyproline du muscle Triceps brachii caput laterale s'est poursuivie en I968-I969 avec un double objectif :

- confirmer la validité des conclusions de la précédente étude sur ce sujet (SELIIER et BoccARD, r969), tout en les complétant par des observations sur les porcs provenant du croisement Piétrain $\times$ Large White,

- en complément à 1'étude d'OLIIVIER (I968), déterminer dans quelle mesure la teneur de ce muscle en hydroxyproline est à prendre en considération dans la recherche de critères objectifs de discrimination entre le "type Piétrain " et le "type Large White ".

Nous nous proposons ici de présenter l'ensemble des données recueillies sur ce caractère et de discuter son utilité pratique dans l'étude de l'hypertrophie musculaire du porc de Piétrain.

\section{MATÉRIEL E'T MÉTHODES}

\section{I. - Matériel animal}

Les données utilisées dans l'étude de Sellier et Boccard (1969) provenaient de 77 porcs de Piétrain et 55 porcs de race Large White, engraissés au domaine de Galle par Avord (Cher) et abattus au cours du second semestre de 1967 . Le poids moyen d'abattage était de 91,7 kg en race de $P i e ́-$ 
train et de $100,7 \mathrm{~kg}$ en race Large White. Ces données sont reprises dans la présente étude et constituent la première série d'abattage (série I967).

Les données de la deuxième série d'abattage (série 1969) ont été obtenues dans le cadre d'une expérience de croisement entre des verrats de Piétrain et des truies de race Large White qui s'est déroulée en I968-I969 sur le même domaine expérimental, dans des conditions d'engraissement analogues (alimentation à volonté). Les porcs Large White, Piétrain et Piétrain $\times$ Large White ont été abattus au poids vif moyen de 95,9, 94,8 et 95,7 $\mathrm{kg}$ respectivement.

Le tableau I donne pour chaque série d'abattage, chaque type génétique et chaque sexe le nombre de carcasses étudiées. Les 633 porcs considérés dans cette étude sont issus de 19 verrats de Piétrain et In verrats Large White.

TABLEAU I

Effectifs contrôlés

\begin{tabular}{|c|c|c|c|c|c|c|}
\hline \multirow{3}{*}{ Série d'abattage } & \multicolumn{6}{|c|}{ Type génétique } \\
\hline & \multicolumn{2}{|c|}{ Piêtrain } & \multicolumn{2}{|c|}{ Piétrain $\times$ Large White } & \multicolumn{2}{|c|}{ Large White } \\
\hline & Mâles castrés & Femelles & Mâles castrés & Femelles & Mâles castrés & Femelles \\
\hline 1967 & 31 & 46 & - & - & 26 & 29 \\
\hline 1969 & 57 & 63 & 130 & 137 & 61 & 53 \\
\hline
\end{tabular}

\section{2. - Mesures effectuées}

Le lendemain de l'abattage, la carcasse (sans tête) a été pesée. La longueur de carcasse (entre l'atlas et le bord antérieur de la symphyse pubienne) et les épaisseurs de lard au niveau de la dernière vertèbre lombaire (rein), de la dernière vertèbre dorsale (dos) et de la dernière vertèbre cervicale (cou) ont été mesurées. La moitié gauche de la carcasse a été ensuite soumise à la découpe parisienne normalisée, dont le principe est décrit par Ollivier (I970). Par ailleurs, au moment de la découpe, le muscle Triceps brachii caput laterale (anconé externe) a été prélevé sur la carcasse et placé dans un congélateur. Le muscle, après enlèvement de ses aponévroses, a été broyé et la détermination du taux d'hydroxyproline a été réalisée sur ce broyat selon la méthode décrite par BocCARD (I968) : il est exprimé par le rapport de l'azote de l'hydroxyproline à l'azote total du muscle.

Les I4 variables suivantes ont été retenues pour l'analyse :

$\mathrm{X}_{1}$ taux d'hydroxyproline du muscle Triceps brachii caput laterale ( $\mathrm{p}$. I ooo).

$\mathrm{X}_{2}$ poids net sans tête $(\mathrm{kg})$.

$\mathrm{X}_{3}$ poids de la tête $(\mathrm{kg})$.

$\mathrm{X}_{4}$ poids du jambon $(\mathrm{kg})$.

$\mathrm{X}_{5}$ poids de la longe $(\mathrm{kg})$.

$\mathrm{X}_{6}$ poids de la poitrine $(\mathrm{kg})$.

$\mathrm{X}_{7}$ poids du hachage $(\mathrm{kg})$.

$\mathrm{X}_{8}$ poids des pieds $(\mathrm{kg})$.

$\mathrm{X}_{9}$ poids de la bardière $(\mathrm{kg})$.

$\mathrm{X}_{10}$ poids de la panne $(\mathrm{kg})$.

$\mathrm{X}_{11}$ longueur de la carcasse (mm).

$\mathrm{X}_{12}$ épaisseur de lard au rein $(\mathrm{mm})$.

$\mathrm{X}_{13}$ épaisseur de lard au dos $(\mathrm{mm})$.

$\mathrm{X}_{14}$ épaisseur de lard au cou ( $\left.\mathrm{mm}\right)$.

Les paramètres statistiques de ces variables dans les cinq échantillons race-série sont donnés au tableau 2. 


\section{3. - Analyse statistique}

Les variables étudiées ont d'abord été corrigées pour les variations de poids vif d'abattage, à partir du calcul effectué intra série et race de la régression des mesures de carcasse sur le poids vif d'abattage. Les variables $\mathrm{X}_{2} \grave{a} \mathrm{X}_{14}$ ont ainsi été ramenées à un poids vif d'abattage de $95 \mathrm{~kg}$. Cette correction n'a pas été appliquée au taux d'hydroxyproline, les coefficients de régression n'étant pas statistiquement significatifs.

TABLEAU 2

Moyennes $(m)$ et écarts-types $(s)$ des échantillons considérés

\begin{tabular}{|c|c|c|c|c|c|c|c|c|c|c|}
\hline \multirow{4}{*}{ Variables } & \multicolumn{10}{|c|}{ Type génétique et série } \\
\hline & \multicolumn{4}{|c|}{ Pictrain } & \multirow{2}{*}{\multicolumn{2}{|c|}{$\frac{P \times L W}{1969}$}} & \multicolumn{4}{|c|}{ Large White } \\
\hline & \multicolumn{2}{|c|}{1967} & \multicolumn{2}{|c|}{1969} & & & \multicolumn{2}{|c|}{1969} & \multicolumn{2}{|c|}{1967} \\
\hline & $m$ & $s$ & $m$ & $s$ & $m$ & $s$ & $m$ & $s$ & $m$ & $s$ \\
\hline Poids d'abattage (kg) & 91,7 & 2,3 & $9 ;, 8$ & 2,8 & 95,7 & 1,9 & 95,9 & 2,3 & 100,7 & 2,7 \\
\hline $\mathrm{X}_{1}$ hydroxyproline $(\%)$ & 3,86 & 0,47 & 3,74 & 0,39 & 3,93 & 0,17 & 4,28 & 0,51 & 4,29 & 0,42 \\
\hline$X_{2}$ poids uet $(\mathrm{kg}) \ldots$ & 67,7 & 2,1 & 69,1 & 2,6 & 69,5 & 2,1 & 68,8 & 2,1 & 73,2 & 2,3 \\
\hline $\mathrm{x}_{3}$ tête $(\mathrm{kg}) \ldots$ & 6,16 & 0,65 & 6,61 & 0,44 & 6,81 & 0,52 & 7,06 & 0,45 & 6,58 & 0,57 \\
\hline$X_{4}$ jambon $(\mathrm{kg})$ & 7,62 & 0,56 & 8,76 & 0,55 & 8,51 & 0,55 & 8,16 & 0,41 & 7,74 & 0,57 \\
\hline $\mathrm{X}_{5}$ longe $(\mathrm{kg})$ & 11,60 & 0,84 & 11,36 & 0,83 & 10,86 & 0,72 & 10,15 & 0,73 & 11,19 & 0,62 \\
\hline $\mathrm{X}_{6}$ poitrine $(\mathrm{kg})$ & 3,42 & 0,30 & 3,62 & 0,38 & 3,71 & 0,36 & 3,79 & 0,38 & 3,67 & 0,38 \\
\hline$X_{z}$ hachage $(\mathrm{kg})$ & 4,48 & 0,31 & 4,50 & 0,33 & 4,59 & 0,31 & 4,48 & 0,28 & 4,83 & 0,36 \\
\hline $\mathbf{X}_{\mathbf{B}}$ pieds $(\mathrm{kg}) \ldots$ & 0,85 & 0,12 & 0,71 & 0,08 & 0,78 & 0,36 & 0,89 & 0,90 & 0,93 & 0,12 \\
\hline $\mathrm{x}_{\mathrm{g}}$ bardière $(\mathrm{kg})$ & 4,69 & 0,92 & 4,53 & 1,04 & 5,24 & 0,87 & 5,95 & 1,02 & 6,69 & 1,00 \\
\hline $\mathrm{X}_{10}$ panne $(\mathrm{kg})$. & 0,82 & 0,22 & 0,75 & 0,27 & 0,78 & 0,34 & 0,79 & 0,24 & 0,88 & 0,20 \\
\hline $\mathrm{X}_{12}$ longueur $(\mathrm{mm})$ & 892 & 31 & 895 & 25 & 927 & 27 & 952 & 25 & 967 & 28 \\
\hline $\mathrm{X}_{12}$ lard rein $(\mathrm{mm})$ & 24,2 & 5,6 & 23,1 & 5,8 & 26,9 & 5,4 & 31,3 & 6,5 & 34,4 & 5,6 \\
\hline $\mathrm{X}_{13}$ lard dos $(\mathrm{mm})$ & 24,2 & 5,4 & 25,1 & 4,9 & 26,9 & 4,8 & 26,7 & 4,8 & 28,4 & 4,7 \\
\hline $\mathrm{X}_{14}$ lard cou $(\mathrm{mm})$. & 40,4 & 5,9 & 42,5 & 6,5 & 46,8 & 6,3 & 48,6 & 7,2 & 50,2 & 5,2 \\
\hline
\end{tabular}

Sur l'ensemble des données et pour les I 4 variables, les effets attachés à chaque niveau des facteurs série d'abattage, race et sexe ont été estimés par la méthode des moindres carrés, une fois le test de l'hypothèse de non-interaction réalisé. Chaque variable a été représentée par le modèle mathématique suivant :

dans lequel :

$$
\mathrm{X}_{i j k l}=\mu+a_{i}+r_{j}+s_{k}+e_{i j k l}
$$

$\mathrm{X}_{i j k l} \quad$ est la variable étudiée.

$\mu \quad$ est une constante.

$a_{i} \quad$ représente l'effet de la série d'abattage $i$ ( 2 niveaux).

$r_{j} \quad$ représente l'effet de la race $j$ (3 niveaux).

$s_{k} \quad$ représente l'effet du sexe $k$ (2 niveaux).

$e_{i j k l}$ est une variable aléatoire de moyenne nulle et de variance $\sigma_{\mathrm{e}}^{2}$.

Pour le taux d'hydroxyproline les 3 groupes génétiques ont été comparés deux à deux à l'aide du test de Duncan et la signification statistique de l'effet d'hétérosis observé a été testée (HARvex, 1960). D'autre part, après correction pour les effets de la série d'abattage et du sexe (données ramenées sur la base des femelles de la série 1969), la normalité des distributions de fréquence de $\mathrm{X}_{1}$ dans les trois groupes a été étudiée en appliquant le test de Kolmogorov-Smirnov (LiLliefors, 1967). 
La non-conformité au modèle ci-dessus pour 7 des $\mathrm{r}_{3}$ autres variables n'a pas permis de réaliser une correction adéquate de toutes les variables et de poursuivre l'analyse avec l'ensemble des données.

La suite de l'analyse, identique à celle appliquée sur les données de la série i967 par SELLIER et BocCARD ( 1969 ), a donc porté sur les données de la $2^{\mathrm{e}}$ série d'abattage relatives aux carcasses des porcs Large White et de Piétrain et corrigées pour l'effet du sexe ; elle comporte deux étapes :

- calcul des corrélations entre les variables étudiées dans les 2 races ;

- analyse discriminante : pour l'établissement de la meilleure fonction linéaire permettant de séparer les deux races à l'aide des variables $\mathrm{X}_{1}, \ldots, \mathrm{X}_{\mathbf{1 4}}$, le choix des variables a été effectué à l'aide de la méthode de sélection progressive mise au point par WAGNER (I965) et basée sur le calcul de la statistique $\mathrm{D}^{2}$ de Mahalanobis selon une méthode décrite par RAo (1952) : la première variable retenue est celle ayant le $\mathrm{D}^{2}$ le plus élevé et à chacun des stades ultérieurs de sélection, la variable retenue est celle qui donne la valeur de $\mathrm{D}^{2}$ la plus élevée avec les variables précédemment retenues. La fonction discriminante tenant compte des six premières variables ainsi sélectionnées a été utilisée dans cette étude.

Tous ces calculs ont été réalisés sur l'ordinateur IBM I620 du Service de Calcul de la Station centrale de Génétique animale.

\section{RÉSULTATS}

Le tableau 3 donne pour les $\mathrm{I}_{4}$ variables les résultats de l'analyse de variance à 3 facteurs avec le test d'interaction globale et, quand l'hypothèse d'additivité est vérifiée, les tests des effets principaux. L'interaction globale est statistiquement significative pour 7 variables; l'analyse des modèles à 2 facteurs montre qu'elle est à attribuer à l'existence d'une interaction série-race pour six d'entre elles : poids net, tête, jambon, poitrine, longueur, épaisseur de lard au cou.

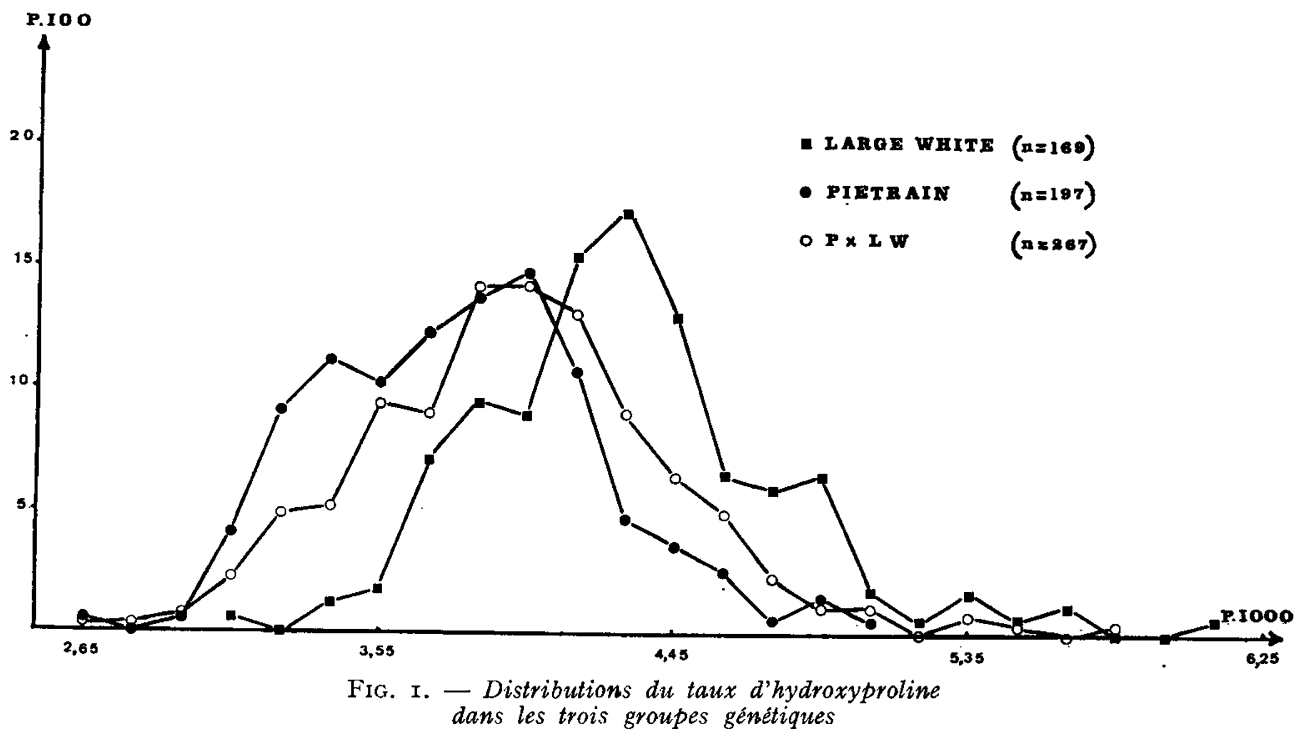

En ce qui concerne le taux d'hydroxyproline du muscle Triceps brachii caput laterale (variable $\mathrm{X}_{1}$ ), l'interaction globale n'est pas significative, ce qui permet l'analyse des effets principaux :

- la différence entre les moyennes des deux séries $(4,048 \pm 0,042$ en I 967 contre $3,980 \pm 0,022$ en I969) n'est pas statistiquement significative, 
P. SELLIER, R. BOCCARD

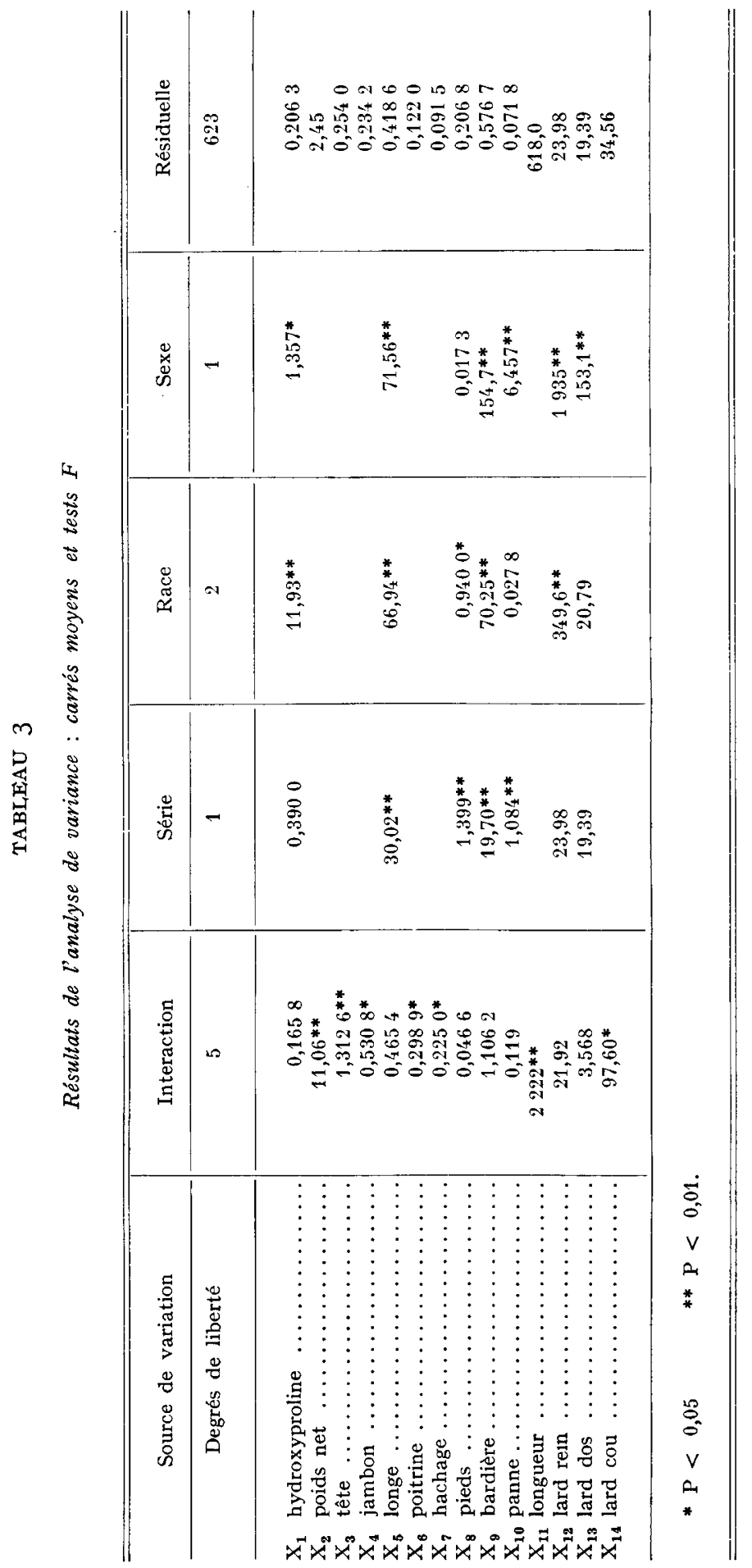




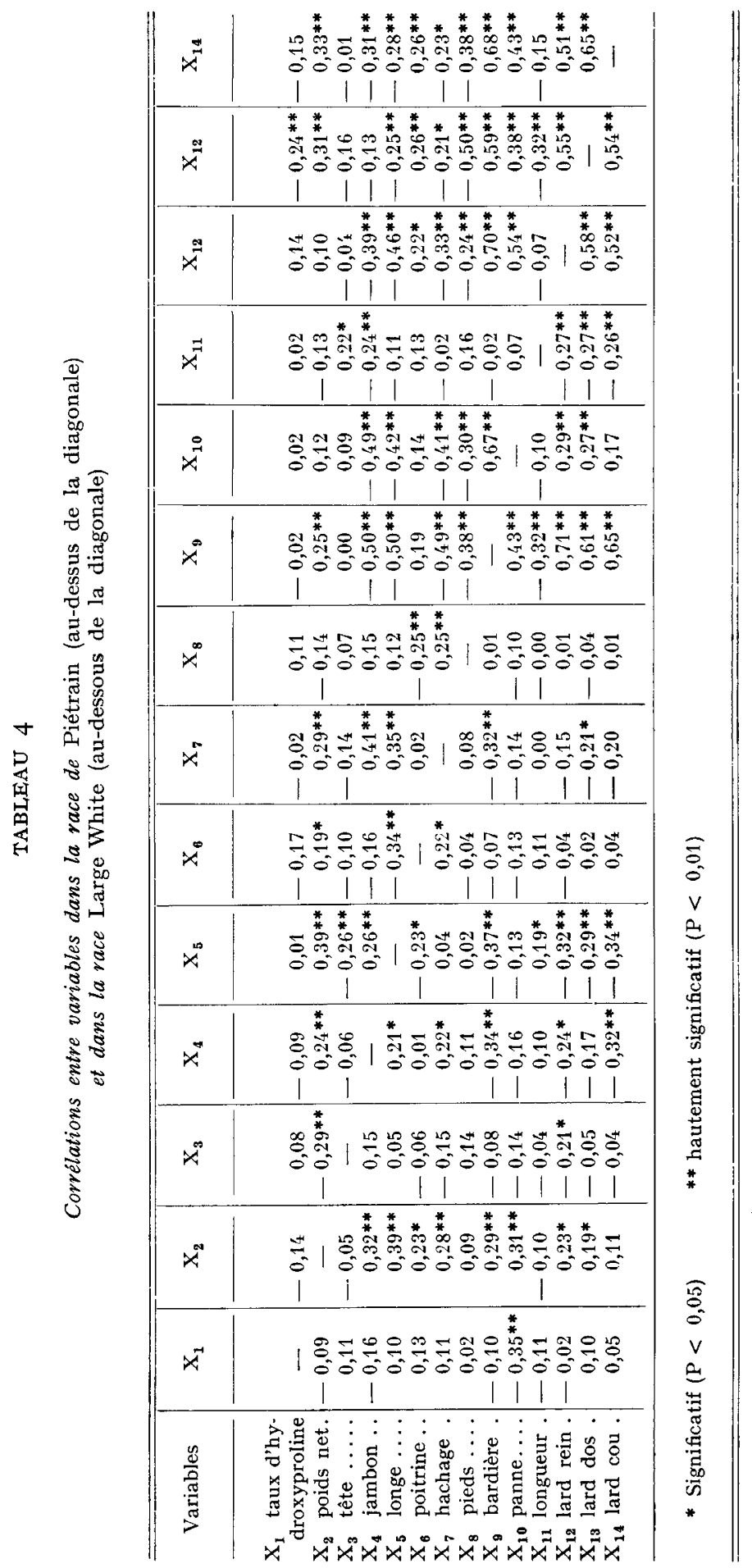


- l'effet du sexe est significatif $(\mathrm{P}<0,05)$, la teneur du muscle en hydroxyproline étant plus faible chez les mâles castrés $(3,967 \pm 0,030)$ que chez les femelles $(4,060 \pm 0,028)$,

- enfin l'effet de la race est hautement significatif $(P<0,0 I): 3,789 \pm 0,033$ chez des porcs de Piétrain, 3,958 $\pm 0,037$ chez les porcs Piétrain $\times$ Large White et 4,295 $\pm 0,036 \mathrm{chez}$ les porcs Large White. Les différences entre ces moyennes prises deux à deux sont hautement significatives $(\mathrm{P}<\mathrm{o}, \mathrm{OI})$. Il apparait d'autre part que dans ce croisement, l'effet d'hétérosis pour le taux d'hydroxyproline est significatif $(\mathrm{P}<0,05)$, les porcs de croisement se rapprochant plus des porcs de Piétrain que des porcs Large White.

Les distributions de fréquence du taux d'hydroxyproline dans les trois groupes génétiques ont été établies (fig. I). L'application du test de Kolmogorov-Smirnov indique qu'il n'y a pas lieu de rejeter (au seuil de 5 p. Ioo) l'hypothèse de normalité de chacune de ces distributions.

Au tableau 4 figurent les valeurs des corrélations entre les variables considérées, à l'intérieur des races Large White et de Piétrain. Les corrélations entre la teneur du muscle en hydroxyproline et les I3 autres variables sont très faibles d'une façon générale ; seuls deux des 26 coefficients de corrélation sont statistiquement différents de 0 : la corrélation est négative entre le taux d'hydroxyproline d'une part, le poids de la panne en race Large White et l'épaisseur de lard au dos en race de Piétrain d'autre part.

TABLEAU 5

Sélection progressive des 6 variables de la meilleure fonction discriminante séparant les échantillons Large White et Piétrain de la série 1969

\begin{tabular}{|c|c|c|c|c|}
\hline $\begin{array}{c}\text { Stade } \\
\text { de sélection } \\
(k)\end{array}$ & Variable retenue & $\begin{array}{l}\text { Valeur du coefficient } \\
\text { de la fonction } \\
\text { discriminante }\end{array}$ & $\mathrm{D}_{2}(k)$ & $\begin{array}{c}\text { Test de } \mathrm{D}_{2}(k) \\
\text { par rapport } \\
\text { à } \mathrm{D}_{2}(k-1)\end{array}$ \\
\hline $\begin{array}{l}4 \\
5 \\
6\end{array}$ & $\left\{\begin{array}{lll}\mathrm{X}_{11} & \text { longueur de carcasse }(\mathrm{mm}) \\
\mathrm{X}_{12} & \text { lard rein }(\mathrm{mm}) \ldots \ldots \ldots \ldots \\
\mathrm{X}_{1} & \text { taux } \ldots \ldots \ldots \ldots \ldots \\
& (\%) \ldots \ldots \ldots \ldots \\
\mathrm{X}_{5} & \text { poids de la longe }(\mathrm{kg}) \ldots \ldots \\
\mathrm{X}_{10} & \text { poids de la panne }(\mathrm{kg}) \ldots \ldots \\
\mathrm{X}_{9} & \text { poids de la bardière }(\mathrm{kg}) \ldots\end{array}\right.$ & $\begin{array}{l}0,126 \\
0,224 \\
3,15 \\
-1,91 \\
-6,21 \\
1,61\end{array}$ & $\begin{array}{l}5,9 \pm * * \\
9,32 * * \\
11,65 * * \\
13,20 * * \\
13,86 * * \\
14,53 * *\end{array}$ & $\begin{array}{l}\text { - } \\
* * \\
* * \\
* * \\
* * \\
* *\end{array}$ \\
\hline
\end{tabular}

** Hautement significatif $(\mathrm{P}<0,01)$.

Le tableau 5 présente les résultats de 1'analyse discriminante réalisée sur les données de la série I969 relatives aux porcs Large White et de Piétrain. On voit que le taux d'hydroxyproline $\mathrm{X}_{1}$ intervient dans la fonction discriminante. En tenant compte seulement des 5 autres variables choisies $\left(\mathrm{X}_{5}, \mathrm{X}_{8}, \mathrm{X}_{10}, \mathrm{X}_{11}, \mathrm{X}_{12}\right)$, le $\mathrm{D}^{2}$ est égal à $\mathrm{I} 2,80$; la prise en considération de la variable $\mathrm{X}_{1}$ apporte une augmentation du $\mathrm{D}^{2}$ hautement significative $(\mathrm{P}<\mathrm{o}, \mathrm{or})$. La fonction $\mathrm{Y}$ choisie permet une bonne 
discrimination entre les échantillons des races Large White et de Piétrain : le chevauchement des deux distributions des valeurs prises par $\mathrm{Y}$ est assez peu important (fig. 2). Les paramètres relatifs à ces distributions sont rapportés au tableau 6 .

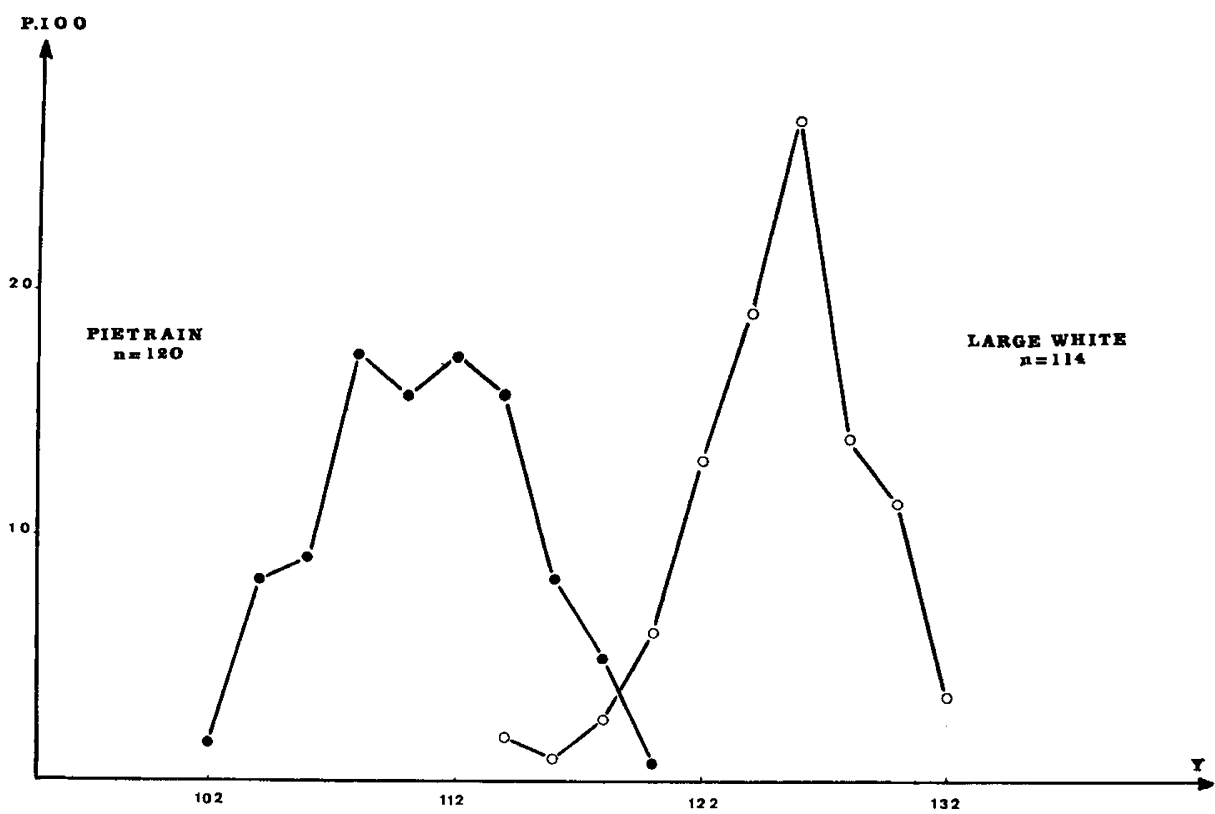

FIG. 2. - Distribution des valeurs de la fonction discriminante dans les échantillons Piétrain et Large White de la deuxième série d'abattage

\section{TABLEAU 6}

Paramètres des distributions de la fonction discriminante $Y$ dans les échantillons Large White et Piétrain de la série 1969

\begin{tabular}{c|c|c|c|c}
\hline Race & Effectif & Moyenne & Variance & Écart-type \\
\hline & & & & \\
\hline Piétrain ....... & 120 & 110,68 & 15,58 & 3,95 \\
Large White..... & 114 & 125,19 & 13,36 & 3,65 \\
\hline
\end{tabular}

La méthode proposée par WAGNER (I965) pour obtenir une indication empirique de l'importance relative dans la fonction discriminante calculée des six variables sélectionnées montre que la part prise par chacune d'elles dans la valeur trouvée pour $\mathrm{D}^{2}$ est de l'ordre de $49 \mathrm{p}$. Ioo pour la longueur de carcasse, I6 p. roo pour le poids de la longe, I2 p. Ioo pour le poids de la bardière et pour le taux d'hydroxyproline, Ir p. Ioo pour l'épaisseur de lard au rein et 0, I $\mathrm{p}$. Ioo pour le poids de la panne.

Désignons par $\mathrm{Y}^{\prime}$ la fonction discriminante tenant compte des 5 variables $\mathrm{X}_{5}, \mathrm{X}_{9}, \mathrm{X}_{10}, \mathrm{X}_{11}$ et $\mathrm{X}_{12}$. Le coefficient de corrélation entre $\mathrm{Y}^{\prime}$ et $\mathrm{X}_{1}$ dans les deux échantillons est très proche de o : - 0,039 en race de Piétrain et $-0,04 \mathrm{I}$ en race Large White. 


\section{DISCUSSION}

Dans la présente étude, la teneur du muscle en hydroxyproline n'a pas été seulement considérée en tant qu'indice de dureté de la viande, comme cela a été le cas chez les Bovins (PARrish et al., I96r ; BOCCARD et al., I967) ; la tendreté de la viande aux poids d'abattage couramment pratiqués, n'a d'ailleurs pas posé à ce jour de problème sérieux chez le Porc. L'objectif poursuivi était aussi d'étudier les relations qui lient ce caractère aux autres mesures de carcasse et d'évaluer sa contribution éventuelle à la définition de critères objectifs de discrimination entre la race de Piétrain et la race Large White.

\section{I. - Observations sur le choix des variables}

Pour l'étude de la teneur du muscle en hydroxyproline, une première raison, d'ordre technique et économique, explique le choix du muscle Triceps brachii caput laterale : son prélèvement, assez facile à effectuer, ne déprécie pas la carcasse. On peut objecter qu'il appartient à une région corporelle - le membre antérieur dont la musculature présente une hypertrophie relativement peu prononcée dans la race de Piétrain : Dumont et Schmirt (I970) ont trouvé que le développement pondéral de ce muscle est très voisin chez les porcs Large White et de Piétrain. Cependant BocCARD (I968), comparant le taux d'hydroxyproline de 7 muscles chez des porcs de ces races, a montré que le muscle Triceps brachii caput laterale est 1'un de ceux pour lesquels la différence entre les deux races est la plus marquée : ceci constitue une seconde raison en faveur du choix qui a été fait. D'une façon générale d'ailleurs, si l'on considère les caractéristiques de différents muscles dans ces deux races, la réduction de leur teneur en hydroxyproline en race de Piétrain n'apparaît pas liée à leur degré d'hypertrophie, comme le suggère le rapprochement des résultats obtenus séparément par Dumont et al. (I969) et par BocCARD (I968) : certains muscles, parmi les plus hypertrophiés en race de Piétrain (Semi tendinosus et Semi membranosus), présentent une réduction du taux d'hydroxyproline relativement peu sensible. Les effectifs impliqués dans les deux études précédentes empêchent d'affirmer l'existence d'une relation entre degré d'hypertrophie et réduction du collagène dans le muscle mais il reste néanmoins que pour l'étude du taux d'hydroxyproline du muscle en tant que critère de discrimination entre les races Large White et de Piétrain, le muscle Triceps brachii caput laterale apparaît comme un choix convenable.

En dehors des trois mesures d'épaisseur de lard dorsal, l'évaluation de la composition corporelle repose sur les proportions des différents morceaux issus d'une découpe commerciale. Une telle découpe est trop globale pour fournir une estimation tout à fait satisfaisante de la composition tissulaire de la carcasse, les variations de poids d'un morceau donné ne reflétant généralement que de façon approchée les différences dans l'importance relative des tissus musculaire, adipeux et osseux. Une dissection de la carcasse ou au moins d'une partie de la carcasse aurait été préférable pour apprécier le degré de développement musculaire des animaux mais elle n'était pas réalisable sur un nombre aussi grand d'animaux dans les conditions de travail d'un abattoir. La précision de l'étude des relations entre taux d'hydroxyproline du muscle et développement musculaire s'en trouve diminuée dans une certaine mesure. 


\section{2. - La teneur du muscle en hydroxyproline}

Les teneurs en hydroxyproline du muscle Triceps brachii caput laterale chez les porcs Large White et de Piétrain, ainsi que la différence de teneur entre ces 2 races, sont beaucoup plus faibles que celles trouvées précédemment par BoccARD (I968) sur le même muscle. L'explication est sans doute à rechercher dans le changement, intervenu entre les deux études, de la technique de prélèvement du muscle. Dans l'étude de BOCCARD (I968), le prélèvement était réalisé par dissection fine et la totalité du muscle, y compris ses parties aponévrotiques, était broyée avant dosage de l'hydroxyproline et de l'azote total. Par la suite le muscle a été prélevé à l'abattoir, nécessairement de façon moins précise et sans que l'intégrité du muscle après prélèvement soit parfaitement garantie; afin d'uniformiser les conditions de mesure, les dosages ont donc porté seulement sur un échantillon correspondant au "cœur » du muscle.

Bien que 1'effet d'hétérosis soit statistiquement significatif pour le taux d'hydroxyproline, il reste faible - de l'ordre de $2 \mathrm{p}$. Ioo - et on peut considérer que sa valeur chez les porcs issus du croisement Piétrain $\times$ Large White est proche de la moyenne des races parentales. Ceci est en accord avec les résultats obtenus sur ce même croisement par JACQUET et OLIIVIER (I97I) qui trouvent que les principaux critères de qualité de la viande (couleur, mesures de $\mathrm{pH}$, pouvoir de rétention d'eau) se comportent dans l'ensemble comme des caractères additifs.

Par ailleurs, dans l'étude de SELLIER et Boccard (I969), la distribution de fréquence du taux d'hydroxyproline présentait une allure bimodale dans l'échantillon Piétrain. Ce résultat est sans doute à attribuer au faible effectif considéré dans cette première étude : pour l'ensemble des deux séries, le taux d'hydroxyproline est distribué normalement dans la race de Piétrain, comme chez les porcs Large White et Piétrain $\times$ Large White.

L'étude de la structure du tissu conjonctif interne dans la section médiane de muscles de porcs Large White et de Piétrain a été entreprise sur le plan histologique par DumonT et SchmitT (I970). Ils trouvent que pour le muscle Triceps brachii caput laterale, non hypertrophié chez les porcs de Piétrain, l'aspect de la trame conjonctive est tout à fait comparable dans les deux races, alors que des différences de répartition du tissu conjonctif interne sont observées dans le cas du muscle Semi membranosus, hypertrophié en race de Piétrain. Il apparaît donc que, pour l'étude comparée du tissu conjonctif du muscle Triceps brachii caput laterale dans les races Large White et de Piétrain, la voie chimique et la voie histologique ne donnent pas des résultats concordants : la réduction de la teneur en hydroxyproline de ce muscle (de l'ordre de I2 p. IOo) chez le porc de Piétrain ne correspond pas à une modification observable de la répartition de la trame conjonctive dans la partie médiane du muscle.

\section{3. - Relation entre taux d'hydroxyproline et développement musculaire}

Les relations entre la réduction de la teneur du muscle en hydroxyproline et le degré d'hypertrophie musculaire sont difficiles à analyser et leur nature dépend du niveau où l'on se place pour les étudier. 
Si l'on compare les deux races Large White et de Piétrain, le développement plus accentué de la musculature des porcs de Piétrain s'accompagne de façon globale d'une réduction de la teneur des muscles en hydroxyproline (BOCCARD, I968). Par contre, si l'on considère les relations entre ces caractères à l'intérieur des deux races, on observe l'indépendance statistique de la teneur en hydroxyproline du muscle étudié ici et des mesures de carcasse liées au développement musculaire : en particulier, il n'y a pas de corrélation significative, dans l'une et l'autre race, entre le taux d'hydroxyproline et la valeur de la fonction $\mathrm{Y}^{\prime}$, considérée comme une mesure individuelle de l'intensité du développement musculaire ; la précédente étude (SELLIER et BocCARD, I969) avait abouti à une conclusion analogue. Dans la mesure où le taux d'hydroxyproline constitue un indice de tendreté de la viande, nos résultats peuvent être rapprochés de ceux obtenus par THORNTON et al. (I968) lors d'une expérience de sélection montante et descendante sur l'épaisseur du lard dorsal dans les races Duroc et Yorkshire; ces auteurs ont observé que la tendreté du muscle Longissimus dorsi tend à être plus grande dans les lignées à forte adiposité, la différence entre la lignée " haute " et la lignée "basse " étant significative pour deux des quatre mesures de tendreté en race Duroc. Cette tendance paraît être en accord avec les corrélations négatives mentionnées plus haut entre le taux d'hydroxyproline du muscle Triceps brachii caput laterale et certains indices d'adiposité, ainsi qu'avec les résultats dela précédente étude (SELLIER et BOCCARD, I969) où des corrélations négatives significatives avaient été observées en race de Piétrain entre le taux d'hydroxyproline de ce même muscle d'une part, le poids de bardière et les trois mesures d'épaisseur du lard dorsal d'autre part.

L'interprétation de l'ensemble de ces résultats n'apparaît pas simple et des études complémentaires seraient nécessaires pour tenter de préciser la nature des relations entre la teneur en collagène d'un certain nombre de muscles et le développement musculaire de 1'animal non seulement intra-race mais aussi au niveau de la comparaison entre la race de Piétrain à musculature hypertrophiée et les autres races.

\section{4. - Contribution apportée par le taux d'hydroxyproline du muscle à la discrimination entre les races Large White et de Piétrain}

Le taux d'hydroxyproline du muscle Triceps brachii caput laterale fait partie des 6 variables retenues dans la meilleure combinaison linéaire séparant les races Large White et de Piétrain. Ceci indique qu'indépendamment des différences de conformation (longueur), d'adiposité (épaisseur de lard au rein) et d'importance de la musculature (poids de la longe), le taux d'hydroxyproline apporte une discrimination additionnelle, hautement significative, entre la race de Piétrain et la race Large White. Notons toutefois que cette variable $n$ 'intervenait pas dans la fonction discriminante précédemment établie par SELIIER et BOCCARD (I969) sur d'autres échantillons des deux races.

Au tableau 7, les résultats de la présente analyse discriminante sont comparés à ceux des analyses réalisées antérieurement (OLLIVIER, I968; SELLIER et BocCARD, I969) sur des échantillons des races Large White et de Piétrain et, à quelques exceptions près, à partir des mêmes variables. On voit que les variables sélectionnées diffèrent sensiblement selon les analyses : la taille réduite des échantillons étudiés explique sans doute en partie ces différences. La longueur de carcasse et le poids de 
TAUX D'HYDROXYPROLINE MUSCULAIRE DU PORC
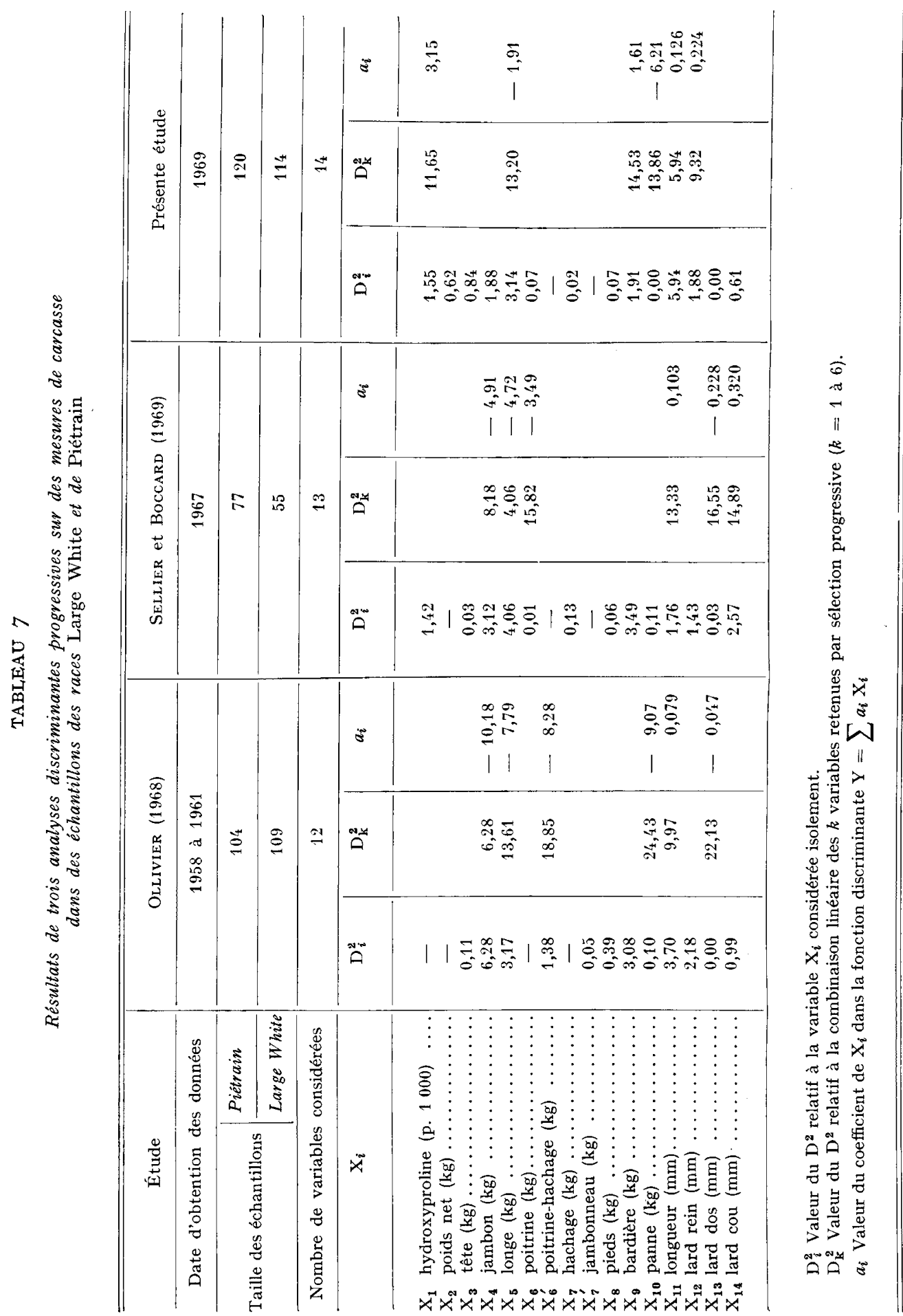
la longe sont des deux seules variables qui figurent dans les trois fonctions discriminantes calculées.

Incidemment on peut remarquer la différence sensible qui existe pour la valeur du $\mathrm{D}^{2}$ entre 1'étude d'Olílvier (I968), concernant des données obtenues dans les stations de contrôle sur la descendance pendant la période I958-I96I, et les deux études ultérieures qui portent sur des animaux nés en I967-I968 et où une ou deux variables supplémentaires (dont le taux d'hydroxyproline) sont considérées. Le faible nombre d'animaux composant les échantillons concernés empêche d'écarter tout à fait l'hypothèse d'une fluctuation purement fortuite due à des erreurs d'échantillonnage pour expliquer cette situation. Mais on peut également penser que l'abaissement $\mathrm{du} \mathrm{D}^{2}$ traduit une diminution de la " distance statistique " entre la population Large White et la population Piétrain au cours de la dernière décennie. L'examen des valeurs $\mathrm{du} \mathrm{D}^{2}$ relatif à chaque variable tend à montrer que le poids du jambon est l'une des variables pour laquelle la réduction du $\mathrm{D}^{2}$ a été la plus nette. Ces dernières conclusion doivent être nuancées par le fait que les conditions expérimentales n'ont pas été rigoureusement comparables : les données utilisées par OLLIVIER (I968) ont été obtenues sur des porcs placés en loges individuelles dans une porcherie fermée, alimentés en régime semi ad libitum et abattus à Ioo $\mathrm{kg}$ alors que les données de l'étude de SELLIER et BOCCARD (I969) et de la présente étude l'ont été sur des porcs engraissés par loges de ro dans des porcheries en semi-plein air, alimentés en régime ad libitum et abattus à des poids moyens inférieurs à roo $\mathrm{kg}$ (sauf pour les porcs Large White de la série I967). Il n'est pas possible d'évaluer la part de la réduction du $\mathrm{D}^{2}$ due globalement à ces différences de conditions de milieu, ni d'analyser l'influence éventuelle de chacune d'elles : il semble de toute façon que la réduction du $\mathrm{D}^{2}$ est trop importante pour s'expliquer entièrement de cette manière et il est permis de penser qu'elle indique dans une certaine mesure un rapprochement des deux populations pour les caractères de conformation et de composition corporelle.

\section{CONCLUSION}

Bien que la prise en considération du taux d'hydroxyproline du muscle Triceps brachii caput laterale constitue un apport utile dans la recherche des meilleurs critères de discrimination entre le type Piétrain, à musculature hypertrophiée, et le type Large White, l'intérêt pratique de ce caractère est limité par le fait qu'aucune relation simple ne semble exister entre ce caractère et la composition corporelle à l'intérieur de chacune des deux races. En particulier il ne semble pas que la teneur de ce muscle en hydroxyproline puisse constituer, en race de Piétrain, une mesure objective et simple du degré d'hypertrophie musculaire d'un animal, tel qu'il a été apprécié dans notre étude. L'étude des relations entre taux d'hydroxyproline et développement musculaire mériterait toutefois d'être reprise en estimant le degré d'hypertrophie musculaire d'un animal de façon plus précise que ne le permettent les résultats d'une découpe commerciale et en prenant en considération d'autres muscles, plus sujets à l'hypertrophie en race de Piétrain. 


\section{REMERCIEMENTS}

Ce texte a été lu par MM. Dumont et Ollivier (I. N. R. A., Jouy-en-Josas). Nous tenons à les remercier de leurs utiles remarques et suggestions.

\section{SUMMARY}

\section{A STUDY OF THE HYDROXYPROLINE CONTENT}

OF ONE MUSCLE AND ITS RELATIONS WITH SOME CARCASS TRAITS

IN THE LARGE WHITE AND PIÉTRAIN BREEDS

In two years (1967 and 1969), I97 Piétrain pigs, I69 Large White pigs and 267 Piétrain $\times$ Large White pigs were slaughtered at $95 \mathrm{~kg}$ liveweight and 13 measurements were taken on the carcass : weight of different cuts, length, backfat thickness at three locations. Also hydroxyproline content in the Triceps brachii caput laterale muscle was determinated : it is expressed as nitrogen of this aminoacid per total nitrogen (in p. I ooo).

Hydroxyproline content is greater $(\mathrm{P}<0.05)$ in females than in castrated males. The differences between the three genetic groups were all highly significant $(\mathrm{P}<0.0 \mathrm{I}): 3.789 \pm 0.033$ in Piétrain pigs, $3.95^{8} \pm 0.037$ in Piétrain $\times$ Large White pigs, $4.295 \pm 0.036$ in Large White pigs. By application of a method of forward selection using the $\mathrm{D}^{2}$ statistic of Mahalanobis, a discriminant analysis was carried out from the I4 variables on the 1969 samples of Piétrain and Large White breeds. Hydroxyproline content is, with carcass lenght, backfat thickness at the last lumbar vertebra and loin weight, one of the selected variables of the best linear function separating the hypertrophied Piétrain breed and the normal Large White breed. However no simple relation seems to exist between hydroxyproline content in the muscle involved and measurements of conformation and body composition within either breed.

\section{RÉFÉRENCES BIBLIOGRAPHIQUES}

Blendi H. M., I965. Ergebnisse der Vollzerlegung in der Fleischleistungsprufüng beim Schwein. Bayer. landw. Jb., 42, 94r-955.

Boccard R., I968. Variation de la teneur en hydroxyproline de muscles de porcs Large White et Piétrain. Ann. Zootech., 17, 7 I-75.

Boccard R., Dumont B.-L., Schmitt O., rg67. Note sur les relations entre la dureté de la viande et les principales caractéristiques du tissu conjonctif. 13th Conference of the European Meat Research Workers, Rotterdam, 20-26 août, 23 p. (ronéoté).

Dumont B.-L., Schmit O., I97o. Anatomie microscopique comparée du tissu musculaire squelettique de porcs Large White et Piétrain. Ann. Génét. Sél. anim., 2, 381-39r.

Dumont B.-L., Schmitt O., Roy G., r 969 . Développement musculaire comparé de porcs $P$ iétrain et Large White. Rec. Méd. vét., 145, 937-947.

HARVEY W. R., r96o. Least-squares analysis of data with unequal subclass numbers. U. S. D. A., ARS $20-8$.

Jacquet B., Ollivier L., I97I. Résultats d'une expérience de croisement Piétrain $\times$ Large White. II. Aptitude du jambon à la transformation en jambon de Paris. Journées de la Recherche porcine en France, 18-19 février, 23-33.

LILlIEFors H. W., 1967. On the Kolmogorov-Smirnov test for normality with mean and variance unknown. Jour. Amer. Stat. Assoc., 62, 399-402.

Mesle L., Giron J., Dumont B.-L., I959. Anatomie et composition chimique du jambon. 5th Conference of the European Meat Research Workers, Paris, 7-I2 septembre, 15 p. (ronéoté).

Ollivier L., I968. Étude du déterminisme héréditaire de l'hypertrophie musculaire du porc de Piétrain. Ann. Zootechn., 17, 393-407. 
Ollivier L., I97o. L'épreuve de la descendance chez le porc Large White français de I953 à I966. I. Analyse de la variation. Ann. Génét. Sél. anim., 2, 31 I-324.

Parrish F.C., Bailey M. E., Haumann M. D., I96I. Hydroxyproline as a measure of beef tenderness. Food. Techn., 16, 68-7I.

RAO C. R., 1952. Advanced statistical methods in biometric research. John Wiley and Sons, New York. Sellier P., Boccard R., r969. Développement musculaire et taux d'hydroxyproline du muscle dans les races porcines Large White et Piétrain. Ann. Génét. Sél. anim., 1, I79 (Abstr.).

Thornton J. W., Alsmeyer R. H., Davey R. J., 1968. Genetic, diet and pig age effects on pork tenderness and palatability. J. Anim. Sci., 27, I229-1232.

WAgner R., r965. Sélection progressive de variables utilisant la statistique $\mathrm{D}^{2}$ de Mahalanobis. Application à la détermination de la meilleure fonction discriminante séparant deux populations de semis de vigne. Ann. Amélior. Plantes, 15, I59-18r. 